that in the first, effect sizes were relatively small, which suggests that this did not represent a major change in the way patients were being managed by Institute staff.

The study highlights the importance of the emergency department as an area of significant psychiatric need. Given the paucity of psychiatrists in Pakistan and in low- and middle-income countries generally, the need will not be met by psychiatrists alone. In Australia and elsewhere, mental health service provision to emergency departments has come under review. Wand \& White (2007) point to the usefulness of the mental health liaison nurse (MHLN) embedded within the emergency department. However, current mental health nurse training in Pakistan is unlikely to provide suitably qualified nursing personnel for such a role. Moreover, given nursing's low status in Pakistan, would such an expert be acceptable to non-psychiatric staff in general hospital emergency departments? The use of MHLNs in emergency departments should not be dismissed, but the challenges should not be underestimated.

The survey is limited by the incompleteness of the diagnostic data. Also, we were unable to determine how many of the records were for multiple attendances of the same patient. Further studies to determine this would provide useful information for service planning.

It would also have been helpful to canvass the response of the emergency department and general hospital staff to the new service, but this was not included in research planning. Canvassing responses retrospectively would probably yield unreliable results. Future work to establish a new service should plan to record the responses of general hospital staff, as well as of consumers and other stakeholders.

\section{References}

Aghanwa, H. (2002) Consultation-liaison psychiatry in Fiji. Pacific Health Dialogue, 9, 21-28.

Bender, K. G. (2001) Social problems in Pakistani psychiatric patients. International Journal of Social Psychiatry, 47(3), 32-41.

Fido, A. A. \& Al Mughaiseeb, A. (1989) Consultation liaison psychiatry in a Kuwaiti general hospital. International Journal of Social Psychiatry, 35, 274-279.

Geller, J. L. (2009) Public psychiatry: the role of the hospital in the care of the mentally ill. In Kaplan \& Sadock's Comprehensive Textbook of Psychiatry (eds B. J. Sadock, V. A. Sadock \& P. Ruiz), pp. 4299-4315. Lippincott Williams \& Wilkins.

Huyse, F. J., Herzog, T. \& Malt, U. F. (1996) International perspectives on consultation-liaison psychiatry. In Textbook of Consultation-Liaison Psychiatry (eds J. R. Rundell \& M. Wise), pp. 228-255. American Psychiatric Press.

Minhas, F. A. \& Nizami, A. T. (2006) Somatoform disorders: perspectives from Pakistan. International Review of Psychiatry, 18, 55-60.

Sensky, T., Greer, S., Cundy, T., et al (1985) Referrals to psychiatrists in a general hospital - comparison of two methods of liaison psychiatry: preliminary communication. Journal of the Royal Society of Medicine, 78, 463-468.

Wand, T. \& White, K. (2007) Examining models of mental health service delivery in the emergency department. Australian and New Zealand Journal of Psychiatry, 41, 784-791.

RESEARCH PAPER

${ }^{1}$ Clinical Lecturer, Greenwood Institute of Child Health, Schoo of Psychology, University of Leicester, Leicester, UK 2Professor, Department of Psychiatry, Institute of Medical Sciences, Banaras Hindu University, Varanasi, India ${ }^{3}$ Professor, University of Leicester Greenwood Institute of Child Health, Leicester, UK, email pv11@le.ac.uk

"Research Assistant, Greenwood Institute of Child Health, School of Psychology, University of Leicester, Leicester, UK

\title{
The effect of aerobic exercise in the maintenance treatment of depression
}

\author{
P. Majumder, ${ }^{1}$ I. Sharma, ${ }^{2}$ P. Vostanis ${ }^{3}$ and C. Bone ${ }^{4}$
}

We investigated the efficacy of aerobic exercise alongside antidepressant medication as an adjuvant maintenance treatment for depression. Fifty patients in remission were randomly assigned to either medication only or medication plus exercise. Assessment of psychopathology was made at 6-weekly intervals (for 24 weeks) using the Hamilton Rating Scale for Depression. The medicationplus-exercise group showed significantly more improvement at 12 and 24 weeks than the medication-only group. This study adds to a growing evidence base that suggests aerobic exercise is worthy of further development in the treatment of depressive disorders.
The role of exercise in treating depression has been studied extensively in recent years. For example, Rethorst et al (2009) carried out a meta-analysis of 58 randomised controlled trials, and found that groups whose treatment incorporated exercise had significantly lower depression scores than controls. De Zeeuw et al (2010) showed that exercise could have preventive potential, as it was found to reduce the likelihood of depression in high-risk employees with sedentary jobs and an inactive lifestyle. Exercise has also been reported to reduce relapse rates. For example, Babyak et al (2000) found that participants with major depressive disorder who used exercise therapy alone had significantly lower relapse rates than those who received medication. 
Despite these promising findings and its low cost, the effectiveness of exercise is yet to be fully addressed in the psychosocial treatment literature or incorporated into mainstream treatment practice. For example, the National Institute for Health and Clinical Excellence (NICE, 2009) recommended 'structured group physical activity' only as a low-intensity psychosocial intervention for subthreshold depressive symptoms, or depression with chronic physical health problems. This may be because many studies have been criticised for their methodological limitations and incomplete literature searches (Rethorst et al, 2009); indeed, the classification and duration of exercise are not specified in the majority of reports. Of further note is that the efficacy of exercise in a maintenance capacity remains largely unexplored. Thus, our rationale was to investigate the role of exercise alongside antidepressant medication as a post-remission maintenance therapy, in terms of reducing both the severity of psychopathology and relapse rates.

\section{Method}

The 50 patients recruited were attending the psychiatric out-patient department of Sir Sunderland Hospital, Banaras Hindu University, India. Selection was made (via referrals from two senior psychiatrists at the hospital) by the researcher who carried out the post-treatment evaluations. The following selection criteria were used:

- age 15-45 years, in order to cope with the physical exercise regime

- a diagnosis of major depressive or dysthymic disorder, according to DSM-IV-TR criteria (American Psychiatric Association, 2000)

- absence of any comorbid psychiatric illness

- no major cardiac or orthopaedic illness that would contraindicate exercise.

Ethical approval was granted by the University's research ethics committee.

After inclusion in the study, patients were prescribed sertraline $(200 \mathrm{mg} /$ day - the maximum permitted daily dose) and alprazolam ( $1 \mathrm{mg} /$ day) for 3-4 weeks in order to achieve remission of clinical depression. Once significant remission - defined as scoring less than 16 on the 21-item Hamilton Rating Scale for Depression (HRSD; Hamilton, 1960) - was obtained, patients were randomly allocated to groups; blinding was not possible, however, as participants were necessarily aware of the treatment group they were in and the researcher who administered exercise therapy also did the follow-up assessment of psychopathology.

\section{Pre-treatment evaluation}

The researcher assessed the following using a semi-structured pro forma:

- sociodemographic and clinical data

- severity of depressive psychopathology (using the HRSD, plus a structured interview guide)
- stressful life events (lifetime and past 3 months), using the 51-item Presumptive Stressful Life Events Scale (PSLES; Singh et al, 1984) (a culturally appropriate instrument)

- melancholic and atypical features of depression (as per DSM-IV-TR)

- baseline heart rate (mean taken from three 1-minute time slots)

- fitness levels using the Harvard Step Test, to monitor compliance with the exercise programme (Brouha, 1943).

\section{Treatment}

Both groups continued to receive sertraline (200 $\mathrm{mg} /$ day) and alprazolam (1 mg/day) and did not take part in any form of counselling or psychotherapy. For the medication-plus-exercise group, participants also received training in daily exercise therapy. That therapy consisted of a 5-minute warm-up, 15 minutes of stationary running, and a 10-minute cool-down, this being convenient and economical for all participants regardless of gender or location and within guidelines on exer cise (Whaley et al, 2006). Participants were also advised not to perform any other exercise beyond usual daily activities. Heart rate recordings were made in order to adjust the intensity of exercise to keep within the target heart rate zone, this being $60-84 \%$ of the maximum achievable heart rate (Whaley et al, 2006); exercise sessions at home were logged and monitored by carers, and the logs were reviewed by the researcher at every follow-up. None of the participants or their carers reported missing any exercise sessions or cutting sessions short. Follow-up was at 6 -weekly intervals for 24 weeks for all participants, and HRSD and PSLES scores were assessed on each occasion. The medication-plus-exercise group also received refresher exercise training and the Harvard Step Test was performed at every follow-up.

\section{Analysis}

Statistical tests used were the chi-square and Student $t$-test. Epi Info 6 software, version 6.04a, was used to analyse the data.

\section{Results}

Although all 50 patients initially agreed to participate, seven did not complete the study (three from the medication-plus-exercise group and four from the medication-only group). Table 1 shows the sample characteristics and features of depression, which were evenly distributed between the groups. Table 2 shows the inter-group comparisons at all 6 -weekly follow-ups. The medication-plus-exercise group showed a greater improvement in HRSD scores (change in score from baseline) than the medication-only group from the first follow-up, at 6 weeks, and this difference became statistically significant at weeks $12(P=0.04, t=2.10)$ and 24 $(P=0.02, t=2.42)$; there was no significant difference between the groups' PSLES scores at any follow-up point. 
Table 1

Baseline characteristics of the sample

\begin{tabular}{|l|l|c|}
\hline Variable & $\begin{array}{l}\text { Medication-only } \\
\text { group }(n=25)\end{array}$ & $\begin{array}{l}\text { Medication- } \\
\text { and-exercise } \\
\text { group }(n=25)\end{array}$ \\
\hline Number (\%) female & $9(60)$ & $6(40)$ \\
\hline Number (\%) male & $16(46)$ & $19(54)$ \\
\hline $\begin{array}{l}\text { Usual degree of physical activity: number (\%) } \\
\text { sedentary }\end{array}$ & $15(65)$ & $8(35)$ \\
light & $9(41)$ & $13(59)$ \\
moderate & $1(20)$ & $4(80)$ \\
\hline Mean (s.d.) age (years) & $29.6(8.2)$ & $30.5(8.9)$ \\
\hline Mean (s.d.) PSLES score for lifetime & $216.2(124.9)$ & $188.0(86.3)$ \\
\hline Mean (s.d.) PSLES score for last 3 months & $105.0(72.6)$ & $107.2(65.3)$ \\
\hline Mean (s.d.) HRSD score & $23.2(4.2)$ & $22.8(3.7)$ \\
\hline Mean (s.d.) number of melancholic features* & $1.2(1.6)$ & $0.7(1.1)$ \\
\hline Mean (s.d.) number of atypical features* & $0.1(0.33)$ & $0.0(0.3)$ \\
\hline
\end{tabular}

HRSD, Hamilton Rating Scale for Depression; PSLES, Presumptive Stressful Life Events Scale.

*Between group comparisons were non-significant.

\section{Table 2}

Mean (s.d.) improvement (difference from baseline) in score on the Hamilton Rating Scale for Depression

\begin{tabular}{|l|l|l|l|l|l|}
\hline Follow-up & $\begin{array}{l}\text { Medication-only } \\
\text { group }\end{array}$ & $\begin{array}{l}\text { Medication-and- } \\
\text { exercise group }\end{array}$ & $t$ & d.f. & $P$ \\
\hline 6 weeks & $2.6(5.3)$ & $4.1(3.4)$ & 1.17 & 46 & $>0.05$ \\
\hline 12 weeks & $2.3(5.2)$ & $5.0(3.4)$ & 2.10 & 45 & 0.04 \\
\hline 18 weeks & $3.0(6.5)$ & $5.6(5.1)$ & 1.45 & 42 & $>0.05$ \\
\hline 24 weeks & $1.0(7.0)$ & $5.6(5.4)$ & 2.42 & 41 & 0.02 \\
\hline
\end{tabular}

Relapse rates were also recorded via subjective reports and as a measured increase of $>50 \%$ from baseline HRSD scores (Neumeister et al, 1997). In total, nine patients relapsed, two from the medication-plus-exercise group (both in week 18) and seven from the medication-only group (one relapse in week 6, two in week 12 and four in week 24). This difference in relapse rates did not reach significance (Fisher's exact test: one-tailed, $P=0.07$; two-tailed, $P=0.14$ )

\section{Discussion}

We found that the maintenance treatment of medication-plus-exercise resulted in significantly lower HRSD scores than medication alone at 12-week and 24-week assessments, as well as a promising reduction of relapse rates. These differences were not considered to be due to extraneous life events, as there was no significant difference between the two groups' PSLES scores. These findings are in line with Rethorst et al's (2009) meta-analysis in terms of the potential for exercise to lower depression scores, and with the finding from Babyak et al (2000) that exercise is effective in reducing relapse. We found no studies, however, on the combined role of exercise and antidepressant medication as a maintenance therapy for depressive disorder. The present findings suggest a potential role for exercise as a protective agent during remission, and one which might reduce long-term cost.

The study was limited in several ways. There is potential for bias via use of a clinician-rated tool such as the HRSD, particularly in an unblinded study. However, to reduce this, the same experienced assessor was used for the pre-intervention and follow-up assessments and a second assessor made random cross-checks at least once for every participant. Assessors completed the rating scales with the participants through discussion. The sample size was limited to 50, which precluded analysis of potential confounds. For example, the medication-only group was randomly allocated a greater proportion of sedentary members from the outset. There was also an unequal proportion of male/female participants; however, this reflected the population of patients within the hospital.

A further limitation was the lack of comparison with an exercise-only group. It is possible that the exercise group may have derived social gain from the increased contact with carers and researchers; interestingly, Babyak et al (2000) raise the point that psychological factors such as proactive agency may be engaged via choosing to exercise.

A further issue for the current study was the drop in the rate of improvement for the medication-only group at week 24 . The reasons for this are unclear; however, such a change dictates that studies with longer periods of assessment are needed to make stronger interpretations. Thus, future studies could benefit from: larger and more representative samples; extended follow-up periods; additional outcome measures (including patient-rated scales); and the inclusion of further control groups, such as exercise only, social contact only, and group versus solitary exercise.

In conclusion, this study adds to the growing evi dence base that suggests exercise is valuable in the treatment of depressive disorders. In particular, we highlight its potential role as a supplementary maintenance treatment during remission. It is worth highlighting that, despite the relatively short time for which the participants exercised, there were measurable benefits. We believe this area of research warrants further attention, particularly as exercise provides a low-cost, flexible and easily implemented treatment with minimal adverse effects, and especially where costly delays in receiving mainstream psychological interventions are commonplace.

\section{References}

American Psychiatric Association (2000) Diagnostic and Statistical Manual of Mental Disorders (4th edn, text revision) (DSM-IV-TR). American Psychiatric Association.

Babyak, M. A., Blumenthal, J. A., Herman, S., et al (2000) Exercise treatment for major depression: maintenance of therapeutic benefit at 10 months. Psychosomatic Medicine, 62, 633-638.

Brouha, L. (1943) The Step Test: a simple method of measuring physical fitness for muscular work in young men. Research Quarterly, $14,31-36$.

De Zeeuw, E., Tak, E., Dusseldorp, E., et al (2010) Workplace exercise intervention to prevent depression: a pilot randomized controlled trial. Mental Health and Physical Activity, 3, 72-77. 
Hamilton, M. A. (1960) Rating scale for depression. Journal of Neurology, Neurosurgery and Psychiatry, 23, 56-61.

National Institute for Health and Clinical Excellence (2009) Depression: The Treatment and Management of Depression in Adults (Update) (CG90). Available at http://www.nice.org.uk/CG90 (accessed 4 February 2013).

Neumeister, A., Praschak-Rieder, N. Besselmann, B et al (1997) Effects of tryptophan depletion on drug-free patients with seasona affective disorder during a stable response to bright light therapy. Archives of General Psychiatry, 54, 133-138.
Rethorst, C. D., Wipfli, B. M. \& Landers, D. M. (2009) The antidepressive effects of exercise: a meta-analysis of randomized trials. Sports Medicine, 39, 491-511.

Singh, G., Kaur, D. \& Kaur, H. (1984) Presumptive Stressful Life Events Scale (PSLES) - a new stressful life events scale for use in India. Indian Journal of Psychiatry, 26, 107-114.

Whaley, M. H., Brubaker, P. H., Otto, R. M., et al (2006) ACSM's Guidelines for Exercise Testing and Prescription (7th edn). Lippincott, Williams \& Wilkins.

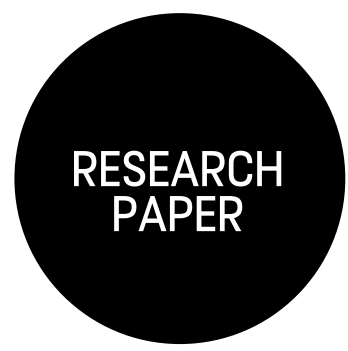

\title{
Scaling up mental healthcare in the Republic of Niger: priorities for and barriers to service improvement
}

\author{
Alison Hwong, ${ }^{1}$ Djibo Maiga Douma, ${ }^{2}$ Soumana Zamo ${ }^{3}$ and Julian Eaton ${ }^{4}$
}

${ }^{1} \mathrm{MD}-\mathrm{PhD}$ Candidate, Harvard Medical School, Boston, MA USA, email Alison_Hwong@hms. harvard.edu

${ }^{2}$ Psychiatrist and Assistant Professor, National Programme in Mental Health, Ministry of Public Health, Niger: Faculty of Health Sciences, Abdou Moun University, Niamey, Niger ${ }^{3}$ Country Representative, CBM Niger Country Coordination Office, Niamey, Niger

${ }_{4}^{4}$ Global Mental Health Advisor, CBM West Africa Regional Office, Lomé, Togo

The authors thank Salifou Badage of the National Menta Health Programme in Niger for his assistance with the in ast Kerim Munir for support and guidance

The CBM International and the Office of Enrichment Programs at Harvard Medical School funded the study
As part of a pilot programme to scale up community mental health services, local health centre directors, community health workers and key informants were interviewed in two neighbouring political districts of Niger. Major priorities for improving services included training staff on the diagnosis and treatment of mental illness, collaborating with traditional healers, educating the community about the origins of psychiatric illness and building infrastructure for medication delivery. Barriers to care included long distances for travel to the nearest hospital and lack of funding for homebased visits by health workers. This study was the first step in Niger's plan to implement the World Health Organization's Mental Health Gap Action Programme (mhGAP) at a national level.

Mental health remains one of the most neglected areas in healthcare in sub-Saharan Africa (World Health Organization, 2011), where the focus of health and development programmes has been on malaria, HIV/AIDS, maternal and child health and malnutrition. Current mental health system reform in Niger plans to use the World Health Organization's Mental Health Gap Action Programme (mhGAP) as a guide to scale up mental health services by integrating them with primary care, employing general healthcare staff in a process of task-shifting (World Health Organization, 2008). This article presents a study that aimed to increase understanding of the context of the reform and barriers to it in two adjacent political districts that are running the programme's pilot phase. The goal of this work is to provide a framework for systematically addressing barriers when scaling up mental health services in Niger (Saraceno et al, 2007; Eaton et al, 2011).

\section{Setting}

Niger, in West Africa, ranks near the bottom of the Human Development Index, which is a combined measure of life expectancy, education levels and standard of living (United Nations Development Programme, 2009). Over $80 \%$ of the country is in the Sahara Desert, which presents unique challenges to healthcare provision. The treatment gap for severe mental disorders is estimated to be over $90 \%$, partly because mental health services and senior professionals are located in central tertiary institutions - primarily at the University Hospital in Niamey, the country's capital (Wang et al, 2007). In the last decade, initial efforts at decentralisation have been made, with 15 psychiatric nurses posted outside of the capital, mostly at regional or district hospitals

Although non-governmental organisations (NGOs) are an important provider of services in Niger, few work in mental health. One exception is the organisation $\mathrm{CBM} / \mathrm{ProDiB}$, which uses a community-based rehabilitation (CBR) model with field workers supported by outreach visits from a psychiatric nurse. In 2010, this organisation partnered with the National Mental Health Programme to develop an implementation strategy for scaling up community-based services at the national level.

To prepare for implementation of the national policy, two districts, Dosso and Dogondoutchi, were chosen for a pilot programme. They are located in the south-western corner of Niger, where most of the population lives, $139 \mathrm{~km}$ and $273 \mathrm{~km}$ east of the capital city of Niamey, respectively. In both regions, subsistence farming involves more than $80 \%$ of the adult population (Système Nationale d'Information Sanitaire, 2007). Table 1 presents health and demographic statistics for the two districts of Niger that are the focus of this study. 